\title{
Slight Skin Lesions in Leprosy and the Importance of their Recognition.
}

\author{
JoHN LOWE.
}

(Reprinted from "Leprosy in India," January, 1933.)

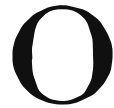

$\mathrm{NE}$ of the great advances in leprosy work in recent years has been in the knowledge of early leprosy. We are now able to recognise the early clinical manifestations of leprosy and are prepared to make a diagnosis without finding the lepra bacillus, which in early cases it may be difficult or impossible to find. This knowledge is now common to most leprosy workers; yet there are some who have failed to acquire this knowledge, and who sometimes write articles which may be misleading. They use such terms as " contacts," " suspected cases," " patient with no definite clinical signs of leprosy," for patients who possibly show definite but slight lesions of neural or even of cutaneous leprosy. They report the frequent finding of bacilli in such " contacts with no clinical signs," and this is often very puzzling to other leprosy workers. Such cases do occasionally occur and have been reported by various workers including the present writer, but they are probably rare. On the other hand, in definite cases of leprosy, it is quite common to find lepra bacilli in apparently unaffected areas of skin. Several such cases have been reported by Lowe and Christian ${ }^{1}$. It must be realised that skin apparently normal on superficial clinical examination will often show slight erythema and thickening on careful examination, and that pathological examination of such skin will often show slight but definite lesions. Also lepra reaction, either occurring naturally or induced by the administration of potassium iodide, will sometimes make these slight or undetectable lesions quite obvious. A failure to realise these facts is leading to confusion in leprosy work, and this is shown in certain articles which we have recently read concerning examination of the blood for lepra bacilli in the diagnosis of leprosy.

The value of blood examination in leprosy is generally agreed to be practically nil. Bacilli can sometimes be found, by the use of a special technique, in the circulating blood of lepers, particularly in advanced cutaneous cases and in cases showing lepra reaction, but this fact has been regarded as being of academic interest and of no value whatever in diagnosis, since bacilli could be found with far greater ease by other methods. 
Markianos', using the thick film method as used in malaria, found that lepra bacilli could be obtained in the thick film only if the puncture was made through definitely leprotic skin, and he considered that the blood simply washed out bacilli lying in the lymph spaces of the skin at the site of puncture. Puncture of normal skin in the same patients showed no bacilli. This is what one would expect. The thick film method is less reliable than the ordinary skin examination for bacilli, by the slit or the clip method.

Two articles we have read recently give a very different account of the results of the thick film method of detecting lepra bacilli.

The first article reports the examination of 26 leper patients by this method. The site at which the blood was taken is not mentioned. Of 4 " skin" cases all showed bacilli. Of 8 "mixed" cases 7 showed bacilli. Of 14 " nerve" cases 10 showed bacilli. In all, positive findings were made in 21 of 26 leprosy cases, and the thick film method is described as a new method of diagnosis of leprosy.

The second article reports even more striking results. Of $38 \mathrm{C}$ cases, 35 showed bacilli in the thick film. Of 14 $\mathrm{N}$ cases, 3 showed bacilli. Of 129 healthy people living in contact with infectious lepers, 6 showed bacilli in the thick film. The film was made by puncturing a finger, and in the leprosy cases, a finger showing no leprous lesion was chosen. The results of nasal smears and examination of the ear lobe are given in each case, a comparison is made, and the thick blood film in leprosy cases is found to give a higher percentage of positive findings than either nasal or skin examination. Of the healthy contacts, ten showed lepra bacilli, six in the blood only, four in the nose and one in the skin as well as the nose. The bacilli found in the thick films are considered to be lepra bacilli circulating free in the blood. The article is illustrated by photomicrographs showing the bacilli in the blood films. One " healthy contact " shows over 50 bacilli in one microscopic field. If this finding is a true one the blood of the patient is teeming with myriads of bacilli, and yet there are no signs of leprosy.

These findings are so much out of keeping with the commonly accepted ideas of leprosy that they are difficult to believe. However, it is no use denying and ignoring these findings. They must be tested experimentally. This we have attempted to do. We examined 50 neural cases by thick film methods and we have not found acid-fast 
bacilli in a single blood film. We have examined 50 cutaneous cases by thick film methods and found acid-fast bacilli in 17 cases. Of these cases 10 showed skin lesions at the site of puncture. The venous blood of these cases was taken and thick films made; bacilli were found in only three of them and then in much smaller number than in the thick film. These three cases were all C3 cases. Thus our conclusions are the same as those of Markianos. For practical purposes bacilli are only found in the thick film when the skin at the site of puncture is leprotic and bacilli are washed out of the skin by the blood. Bacilli are rarely found in the circulating blood.

How then can we explain the findings of the two articles quoted? We are forced reluctantly to adopt the view that the authors of these articles failed to realise that the skin from which the blood was taken was leprotic. The cutaneous cases probably had a generalised cutaneous leprosy; some of the neural cases had some, possibly slight, leprotic changes in the skin, and some of the so-called healthy contacts were definite cases of leprosy with cutaneous lesions, possibly quite slight or even undetectable without very careful examination. This seems to be rather a sweeping assumption to make, but there seems to be no other possible explanation. This view receives considerable support from a careful examination of the data given in the articles. Several of the neural cases are reported as showing bacilli in the nasal mucous membrane and the skin of the ear lobe. If very careful examination of the skin at the site of puncture for the blood film had been made, we think that this skin too would probably have shown slight lesions and acid-fast bacilli. The same remark can also be made regarding the healthy contacts, for several of them showed bacilli in the nose and in the skin of the ear lobe.

Another way in which the failure to recognise the slighter lesions of leprosy may lead to confusion is here illustrated. The following is quoted from the description of leprosy in a standard text-book of medicine in its 1930 edition $^{3}$. After classifying cases as " nodular" and " anæsthetic" the writer describes the initial symptoms in the two forms as follows :-

\section{" Nodular Leprosy."}

Prodromata are usually marked; irregular rises of temperature, rigors, lassitude, drowsiness, diarrhæa and profuse sweating. These may be slight and not absolutely pathognomonic of the disease. The first positive evidence is the primary exanthem or macular stage. This may then 
disappear leaving only a slight brownish discolouration. Fever, however, recurs with a fresh eruption, and after one or two recurrences the characteristic nodules or tubercles appear.

\section{Anaesthetic Leprosy.}

Prodromata are less marked; feelings of depression, chilliness and malaise with shooting pains in the ulnar and peroneal nerves may be encountered.

To an Indian leprosy worker this account is most astonishing. It does not fit in at all with one's practical experience of the initial stages of leprosy. It reads very much more like a description of lepra reaction, and in India reaction is rarely seen at the onset of the disease but usually months or years after the initial symptoms. The earliest stages of leprosy are usually very insidious and not marked by prodromata such as are described above.

There seem to be two possible explanations of this difference of opinion. The first is that leprosy in some countries shows itself in a much more acute form than in India. The second is that the earlier stages and slight lesions are overlooked or ignored and that, until reaction occurs, a diagnosis is not made. Possibly both these factors help to cause this difference of opinion, but on the whole we think that the second factor is much more important. We remember that the Philippine workers used to consider that the slight cases with only one or a few macules, which are so common in India, were extremely uncommon in their country. Further investigation has shown that such cases are not so uncommon as was previously supposed.

The point we would emphasise is that skin which appears normal on casual inspection, may reveal clinical evidence of leprosy on careful examination, and that even if there is little clinical evidence of leprosy, bacteriological and pathological examination may show a very definite leprous lesion containing acid-fast bacilli. The commonest cutaneous lesion of leprosy is not the nodule or the marked thickening, which are detected at a glance, but the very slight erythema and thickening, which are only detected by careful examination.

\section{REFERENCES.}

(1) Love, John, and Christian, E. B.-“ Bacteriological Examination in Leprosy." Ind. Journ. Med. Res., Vol. xix, No. 3, 1932, pp. 867-872

(2) Markianos, J._- "Search for Lepra Bacilli in a Thick Drop." Bull. Soc. Path. Exot., Vol. xxiv, No. 3, 1930, pp. 172-173.

(3) Carmichael Low, G._- "Text Book of the Practice of Medicine." Edited by Frederick Price, 1930. 